\author{
Surg Capt WP THERGAONKAR*, Maj Gen DP ACHAR+
}

\begin{abstract}
The tuberculosis situation in the country is a matter of great concern since the disease has not been contained. The problem has been further compounded by the emerging problem of HIV infection in the country together with development of multi-resistant tubercle bacilli. There is, therefore, a need to change our National Tuberculosis Control Strategy without disturbing the basic infrastructure of the National Tuberculosis Programme. Changes such as reinforcement of the District Tuberculosis Centre, HIV and drug sensitivity testing, giving up of long term chemotherapy, BCG vaccination policy, chemoprophylaxis policy and involvement of Non-Governmental Organisations and general practitioners are suggested.
\end{abstract}

MJAFI 1995; $51: 47-50$

KEY WORDS : Human immunodeficiency virus; Tuberculosis.

D espite existence of the National Tuberculosis Programme for over 3 decades, the situation of tuberculosis is grim in the country. Tuberculosis is responsible for 500,000 deaths annually in India [1]. According to an estimate, there were 10 million radiologically active cases of pulmonary tuberculosis in India in 1981 of which $2.5 \mathrm{mil}$ lion were infectious and as many as $50 \%$ people in India are infected by tubercle bacilli although they may appear healthy [2]. As per the estimates, there are more than 4 million people, mostly in developing countries, who have been infected with both HIV and tuberculosis [3]. HIV infection, by progressively impairing cell mediated immunity, appears to be the highest risk factor for reactivation of tuberculosis into an active disease [4]. It is a well known fact that HIV infection is spreading unabated throughout the length and breadth of the country and as per the estimates, by $2000 A D, 400,000$ HIV infections will have occurred in the country. Thus tuberculosis can be considered as the most im- portant candidate as an opportunistic infection in HIV infected individuals in the country. There is every possibility of the problem being compounded by infections or reinfections occurring with multi-drug resistant strains of tubercule bacilli [5]. As it is, the problem of drug resistant strains throughout the country remains unmapped and any emergence of such new strains may lead to their spread both among HIV infected persons as well as the general population. In such a complicated scenario it has become imperative to take a second look at our National Tuberculosis Programme and make changes consistent with the emerging problems. Suggested changes are as following :

\section{Organsiation of the programme}

Organisational set up of the National Tuberculosis programme should essentially remain the same with the District Tuberculosis Programme (DTP) as its functional arm. The District Tuberculosis Centre (DTC) needs to be provided with additional facilities such as

*Joint Director, Medical Sorvices (Health) and Sr Adviser in Preventive Medicine. Office of the DGMS (Navy), "A" wing. Sena Bhawan; + Additional Diructor General, Armed Forces Medical Services. (E \&S). " $M^{n}$ Block: New Delhi-110 011. 
those for culture, drug sensitivity testing and HIV testing. The former due to ever increasing number of resistant strains and latter due to the peculiar relationship between HIV/AIDS and tuberculosis. Training, by the National Tuberculosis Institute (NTI) Bangalore, of District Tuberculosis officers and laboratory assistants in the techniques of drug resistance testing and HIV testing will obviate the need to post a microbiologist on the staff of the DTC. We may however need to post an additional laboratory assistant at the DTC for these additional commitments.

\section{Case finding activities}

These should also essentially remain the same with emphasis on sputum examination of the patients with symptoms referable to the chest, since, even with HIV infection in an early stage when cell mediated immunity is only partially compromised, typical post primary tuberculosis with upper lobe infiltrates and cavitation is seen and may often be due to reactivation of latent infection [6]. By and large this should hold true in India, given the prevalence of pulmonary infection by tubercle bacilli. The health workers also need to be made aware of extra pulmonary tuberculosis occurring among the HIV infected individuals. Tubercular lymphadenitis is the most frequent form of extrapulmonary tuberculosis and should arouse suspicion amongst the workers.

In sputum negative cases, repeat sputum microscopy followed by chest radiography at the DTC may have to be carried out in some cases which will only be possible when we reinforce laboratory facilities at the DTC.

\section{Treatment}

In view of the emergence of multidrug resistant tubercle bacilli as well as the unabated spread of HIV infection, time has now come for us to rethink about our conventional long term drug regimens i.e. $R 1$ ( $2 \mathrm{STH} / 10 \mathrm{TH}$ ) and R2 (12 TH) which are combinations of isoniazid with thiacetazone and streptomycin. R3 to R5 regimens have since been given up. It is now strongly felt that we should replace these conventional long term regimens by short course chemotherapy. Evaluation of the International Union against Tuberculosis and Lung Diseases (UVALTD) assisted National Tuberculosis Control Programme, has shown that a well managed short course chemotherapy is highly cost effective in a variety of often difficult conditions [7]. In the Indian context where HIV infection is spreading and where it is not yet possible to screen every case of tuberculosis, the best short course chemotherapeutic regimen will be supervised intensive phase of daily isoniazid, rifampicin, pyrazinamide and ethambutol for two months followed by four months of thrice weekly isoniazid and rifampicin or 6 months of daily isoniazid and ethambutol. Replacement of streptomycin by ethambutol as the fourth drug during the initial phases will prevent the potential transmission of HIV and other blood borne pathogens by contaminated needles and syringes. Use of thiacetazone should be altogether given up because of the risk of severe adverse reactions when thiacetazone is used in HIV infected persons [3]. Short course chemotherapy in all tuberculosis patients will also ensure better compliance and less drug default. The patients who do not respond to such a standard course of therapy should be sent to the DTC or nearest medical college where, after necessary investigations, patients could be placed on supervised indoor therapy.

\section{BCG vaccination}

BCG vaccination has till date remained a contentious issue. This is because of the variable results of the tuberculosis prevention trials all over the world. The famous Chingelput trial in South India revealed that BCG vaccination afforded no protection at all against adult forms of pulmonary tuberculosis [8]. However, as BCG is effective in preventing the severe forms of childhood tuberculosis such as TB meningitis and military tuberculosis, WHO has recommended that BCG vaccine be administered as early as possible to the children in countries where the disease has a high prevalence [9]. In our 
country BCG vaccination is, therefore, offered to all infants preferably at birth under the aegis of UIP as per the National Immunization schedule. In patients with full blown AIDS where immune mechanism becomes paralyzed, severe forms of tuberculosis such as miliary tuberculosis or tubercular meningitis are likely to develop as opportunistic diseases and as such it will be only logical to consider that BCG vaccination before the exposure of the individual to HIV infection will help prevent these dreadful complications. It is, therefore, strongly felt that we should revaccinate children with BCG in school at 12 to 15 years of age which is much before their likely exposure to HIV through sexual contact. Re-vaccination could be arranged throughout the district by the DCT with the help of BCG Team which should be revived as a part of the District Tuberculosis Programme (DTP).

\section{Preventive chemoprophylaxis for HIV positive individuals}

Hitherto chemoprophylaxis was not a part of National Tuberculosis Programme. However, considering the ever-increasing burden of HIV associated tuberculosis, chemoprophylaxis now needs to be considered as an essential intervention and needs to be intergrated with the National Tuberculosis Programme. In the USA, as per the current recommendations, isonex is given daily as preventive therapy for 12 months for HIV positive individuals with more than $5 \mathrm{~mm}$ tuberculin test [10]. In our situation, we may consider $10 \mathrm{~mm}$ tuberculin test as a positive test with $1 \mathrm{TU}$ and give chemoprophylaxis with isonex to HIV positive individuals showing positive tuberculin test. Alternatively, we may also consider giving a short course of rifampicin alone or rifampicinpyrazinamide combination for two months [11]. Drugs could be issued under the aegis of National Tuberculosis Programme. It will, therefore, be necessary to carry out tuberculin tests on all the HIV carriers who are asymptomatic. This will, however, be a big effort. We may also, therefore, consider blanket chemo- prophylax is for all HIV carriers without prior tuberculin testing, in view of high prevalence of tuberculosis infection in our country and difficulties involved in tuberculin testing.

\section{Involvement of general practitioners}

There is an urgent need to involve number of general practitioners and laboratories within the district in the DTP. Depending upon the facilities available with the practitioners, they could be given the status of RC (Reporting Centre). They can make sputum smears and send them to the nearest Microscopic Centre (MC) where facilities for staining and microscopic examination of sputum are available. Patients can be referred to X-ray centres for radiological examination. In return, for every case detected by them, the general practitioner should be supplied with free anti-tubercular drugs which they should under their own supervision, administer to the patients. For maximizing case detection all the general practitioners involved must be motivated to get the sputum examination done for all the patients with symptoms referable to chest. They should also be motivated to inform details of confirmed cases of tuberculosis being managed by them to the DTP for inclusion in the district tuberculosis case index. They should also develop a high index of suspicion for the possible coexisting HIV infection.

\section{Involvement of voluntary organisations/ non governmental organizations (NGOs)}

Voluntary and NGOs such as Rotary Club, Lions Club, Youth Club, Mahila Mandal, etc., should be involved in the programme for health education where emphasis should be on self-reporting by symptomatic individuals on preventing drug default as well as for BCG vaccination.

\section{Legislation pertaining to tuberculosis}

Time has now come for an appropriate legislation whereby we make it compulsory for every practitioner of medicine to notify all cases of confirmed tuberculosis to the DTC. We should also have a legislation as per which every case of suspected drug resistance 
is compulsorily admitted, isolated and appropriately treated under expert supervision. This will help prevent the spread of drug resistant bacilli in the community.

\section{Training}

The key programme workers of the DTP should continue to be trained by the NTI Bangalore as before. There is however an urgent need to orient them for the HIV - AIDS Tuberculosis challenge and this aspect should be included in their basic/refresher training.

\section{Research}

Research needs to be conducted in the country in the following areas :

a) To develop and test new methods for diagnosis of tuberculosis such as ELISA, endoscopic bronchoalveolar lavage, transbronchial biopsy, polymerase chain reaction, nucleic acid hybridization processes etc.

b) To find out the efficacy of primary or post-primary forms of tuberculosis in different parts of the country.

c) To develop a more effective immunization against tuberculosis.

d) To find out the prevalence of drug resistance in different parts of the country.

e) To develop new drugs and carry out their trials.

f) To develop an effective system for detection of drug defaulters. g) To find ways and means to involve per, ple in the programme implementation.

\section{REFERENCES}

1. Vishwanathan R, Datey KK, Shah SJ. API Text Book of Medicine. Bombay : Association of Physicians of India. 1979.

2. Suri AK. National Tuberculosis Control Programme, NIHFW.Delhi National Health Programme Series, No.10.

3. Raviglione MC. Narain P. Koch A. HIV Associated Tuberculosis in Developing Countries : Clinical features, diagnosis and treatment. Bull World Health Organ 1992; 70 : 515-26.

4. Riedler HL. Epidemiology of Tuberculosis. Epidemiol Rev. 1989; 11 : 79-80.

5. Pavri KM. "Partners in Crime" : Tuberculosis and HIV/AIDS. CARC Calling 1993; $6: 7$.

6. Harriers AD. Tuberculosis and human immunideficiecy virus infection in developing countries. Lancet 1990; 335 : 387-90.

7. Tuberculosis Control and research strategies for the 1990s : Memorandum from a WHO meeting Bull World Health Organ 1992; 70 : 17-21.

8. Tuberculosis Prevention trial, Madras. Indian J Med Res 1979; 70 : 349-63.

9. BCG Vaccination Policies. WHO Technical Report Series, 652; 1980 : 10-17.

10. Centre for Disease Control, Tuberculosis and Human Immuno deficiency virus infection, Recommendations of Advisory Committee for the Elimination of Tuberculosis (ACET), Morbidity and Mortality Weekly Report. 1989; 38 : 236-50.

11. Clermont $\mathrm{H}$. Tolerance of Short course tuberculosis chemoprophylaxis in HIV infected individuals. Proccedigs of VII International conference on AIDS, 1991 Jun 16-20. Florence Italy, (Abstract) WB 2023 : 187. 